\title{
Women's Autonomy in Household Purchasing Decision Making in Sri Lanka: An Application of Multivariate Baseline-Category Logit Model
}

\author{
D. G. S. Chandradasa ${ }^{*}$, N. Withanage ${ }^{1 \#, ~ A . ~ S . ~ A n a n d a ~}{ }^{2 \# \#}$ \\ ${ }^{1}$ Department of Statistics, Faculty of Applied Sciences, University of Sri Jayewardenepura, Nugegoda, Sri Lanka \\ ${ }^{2}$ Department of Social Sciences, Faculty of Social Sciences and Languages, Sabaragamuwa University of Sri Lanka, Belihuloya, Sri Lanka \\ Email: *csachiki@gmail.com, " niroshan@sjp.ac.lk, "\#arathananda@ssl.sab.ac.lk
}

How to cite this paper: Chandradasa, D. G. S., Withanage, N., \& Ananda, A. S. (2021). Women's Autonomy in Household Purchasing Decision Making in Sri Lanka: An Application of Multivariate Baseline-Category Logit Model. Advances in Applied Sociology, 11, 84-98.

https://doi.org/10.4236/aasoci.2021.112007

Received: January 16, 2021

Accepted: February 16, 2021

Published: February 19, 2021

Copyright (อ 2021 by author(s) and Scientific Research Publishing Inc. This work is licensed under the Creative Commons Attribution International License (CC BY 4.0).

http://creativecommons.org/licenses/by/4.0/

\begin{abstract}
As many researches on gender and household purchasing decision making are sociological as well as descriptive, this attempt tries to fill the gap with applying of statistical model in to the same context. This is basically an inductive approach that generates data, applies joint modeling and moves forward in sociological interpretation. The data has been generated in an Island-wide survey consisting 28,800 household units in Sri Lanka in 2016. Generalized linear mixed approach was applied to baseline-category logit models to identify the factors associated with women's autonomy in decision making on daily household purchases and major household purchases. This research comes up to the conclusions that women's age, education status of the women and spouses, number of children, residence area and economic status in the family emerged as important factors associated with women's autonomy in decision making. Increased age, well paid employment and having many living children are all positively associated with women's autonomy in decision making in both daily household purchases and major household purchases. Women from rural and estate areas are less likely be autonomous in decision making than the women from urban areas. Highly educated women are more likely to make decisions jointly while the women who less attended formal education are further likely to make decisions unaccompanied.
\end{abstract}

\section{Keywords}

Women's Autonomy, Baseline Category Logit Model, Generalized Linear Mixed Models, Household Purchases

\section{Introduction}

The role of gender and household decision making is a common and well-discussed 
topic in all aspects of family studies. Within this context, the household purchasing may be rather significant in terms of marking the female position of society. The general or sometimes the traditional notion on this factor is the male dominances in purchasing durable commodities and services such as cars, family insurance etc., female supremacies regarding kitchen-based utensils while both are responsible on deciding children's schooling, holiday destinations etc. (Davis \& Rigaux, 1974). However, some have emphasized other variables such as ethnicity and race (Abraham, 1995; Bhopal, 2019), social class (McGinn \& Oh, 2017), religion (PRC, 2016), education status or income level (Smyth, 2007) etc. may be predominant in changing gender roles that characterize purchasing decisions in household level. When it is considering to the Sri Lankan society, the gender role in the same framework shows sensitivity on its sector-wise demarcations such as Urban, Rural and Estate. According to the most recent island wide official survey of population and housing conducted in 2012, Sri Lanka's sector-wise population reports such as Urban 18.2 per cent, Rural 77.4 per cent and Estate 4.4 per cent (CBSL, 2018). According to the recent statistics, the population of females is 11.1 Million while male population records 10.4 Million against to the total of 21.5 Million peoples in Sri Lanka of the same year, which is a whopping seven hundred thousand (700.000) more women than men (http://www.pulse.lk). Since the population of Rural and Estate sectors exceeds 80 per cent of the total population, this research may be vital to indulgent the women's capacity of household purchasing decision.

The Sri Lankan woman has been shaped by patriarchal values which have been embedded into its culture through traditional, colonial and post-independence societies in general. However, women in Sri Lanka are placed in a relatively favorable position when compared to women in other South Asian countries (UNDP, 2016), according to standards set by the UN Convention on the Elimination of All Forms of Discrimination against Women (CEDAW) and some of the Millennium Development Goals (MDGs). They also enjoy a high life expectancy of 74 years, nearly universal literacy, and access to economic opportunities, which are almost unmatched by the rest of the subcontinent (Asian Development Bank, 2019).

To understand a woman's status in the society, it is important to inspect their role not only in their public participation, but also in their domestic households as well. By custom, the Sri Lankan woman lives in a patriarchal social system. Therefore, just like in other similar patriarchal societies, in Sri Lanka, men have greater power in making decisions within a household, simply by the virtue of gender. In a household, there will be many decisions made as a single unit. In such cases, both the husband and wife must have a say in them. But in some cases, gender-based power inequalities in decision making can lead to a restriction in open communication between partners, which can then lead to complications. As an example, if a woman has no autonomy over her health care decisions, it can contribute to poor health outcomes. When compared to western 
countries, this situation seems to be slightly prominent in South Asia, where girls and women are greatly excluded from making decisions and have limited access to and control over resources (Jejeebhoy \& Sathar, 2001). Hence, women's autonomy has been a concentrated subject matter. Therefore, it is important to identify and understand the determinants of women's decision making autonomy. Data from two Asian countries, Bangladesh and Nepal, show that variables such as increased age, higher level of education, residency in urban areas, paid employment, household wealth, and greater number of living children show a positive and significant relationship with greater autonomy (Haque et al., 2012; Acharya et al., 2010).

This research has been focused two different aspects of the Sri Lankan women's decision making on: 1) major household purchases decision making, and 2) daily household purchases decision making, and the socio-demographic factors which affect them. A very similar study to this research problem has been conducted by Acharya et al. (2010) in Nepal using the Nepal Demographic and Health Survey (NDHS) - 2006 data. There the associations between the household purchases decision makings (responses) and socio-demographic variables were identified applying multivariate logistic regression for each response separately. Similar studies can be found by Senarath \& Gunawardena (2009), Mujahid et al. (1991), Boateng et al. (2014), Nigatu et al. (2014) and many more. However, in the analysis they all considered responses are independent and separate models for each of responses was fitted. If a married woman is asked about the person (respondent, her husband or partner, together) who takes the decision on: 1) major household purchases, and 2) daily household purchases, the outcomes are likely to be correlated. Hence, in the analysis of finding the relationship between the above responses and socio-demographic variables, the correlation between the responses should be considered into account for valid inferences. In this research we propose a joint modelling approach to investigate the relationship between the correlated responses and predictors via Multivariate Baseline Multicategory Logit Model.

The study made by Yusof (2015) has compared the women's ability to have control over household finance with the use of several socio-cultural variables. Although, this study directly focused on household purchasing, it is also highly attached with common issues of gender, finance and household expenditure. It emphasizes that Malaysian-Chinese families are yet rely on traditional patriarchal values of household finance while modern education has been remarkably effects positively that changed women's autonomy within home premises among all ethnic groups in the urban sector. Yusof \& Duasa (2010) argue again in their study on 'expenditure pattern of married men and women in Malaysia' that women's autonomy is significant in everyday household expenditure and men are dominant at the time of spending high-cost purchasing. However, the joint decision is noteworthy of family investment in business. Xia et al. (2006) claim for appearing of more egalitarian characteristics among husbands and wives 
of Singaporean families in purchase decision-making process compare to other Eastern countries and sometimes that of United States of America. The research has paid exceptional attention on gendered stereotypy of consumer items rather than income or educational variables. Thus, the study is proven the integral relationship of conventional ideologies of gender and symbolic consumer items among spouses emphasizing things such as wife's interesting (rather than domain) in purchasing wife clothing, cleaning supplies, children's wear etc. and husband's favor in electric/electronic items, wine/alcohol, insurance and so on. Moreover, the furniture, eating out, education, entertainment etc. have been laden into the syncretic purchasing decision-making category of the same research.

\section{Statistical Method}

This article adopts quantitative research method to identify the factors influencing the women's autonomy in decision making on major household purchasing and daily household purchasing.

\subsection{Sampling}

The data used for this study were obtained from the Sri Lanka Demographic and Health Survey (SLDHS) 2016. Demographic and health surveys, also known as DHS programs, are national representative population-based household surveys, which provide accurate and internationally comparable data on health indicators in developing countries. They collect and disseminate data on areas such as fertility, family planning, maternal and child health, gender, HIV/AIDS, malaria, nutrition, etc. DHS surveys are part of the world-wide DHS project, intending to observe and improve population health (The DHS program, 2016).

The SLDHS 2016 was conducted and compiled by the Department of Census and Statistics of Sri Lanka. This survey has used a two-stage stratified sampling design, where the population was stratified by district at the first level. As the second level, the district was stratified as urban, rural and estate sectors. 2500 census blocks were selected at the first stage and 12 housing units were selected from each census block at the second stage. A total of 28,800 housing units have been selected by this procedure. Data was collected through the 2016 SLDHS questionnaire using the Computer Assisted Personal Interview (CAPI) method. This questionnaire has been adjusted from the standard ICF DHS core questionnaires by including a multitude of country-specific questions, in order to make the study more relevant to Sri Lanka. These adjustments were mainly done for the questions related to health to reflect the health issues relevant to Sri Lanka (The DHS Program, 2016).

Under the DHS 2016, 28,720 housing units were selected and 27,210 individuals were successfully interviewed. From these individuals, 18,510 women were selected as eligible women (ever-married women in the reproductive age 15 - 49) to complete the women's data section of the questionnaire, out of which 18,302 
were successfully interviewed. The data obtained from them have been used to conduct this study.

\subsection{Responses and Predictors}

The DHS includes four areas regarding decision making, which are on healthcare, major household purchases, daily household purchases and family visits. As the questions of interest for this study, two aspects (responses) regarding household purchasing were selected: 1) who makes decisions on major household purchasing (Response 1), and 2) who makes decisions on daily household purchasing (Response 2). Each response corresponded to four outcomes: 1) Respondent herself, 2) Respondent's husband, 3) Respondent and her husband jointly and 4) Someone else. However, very few respondents (less than 1 percent) had the choice of last category ("someone else"). Hence, to avoid the problems related data scarcity while cross tabulation and modelling, only individuals who responded out of "Respondent herself", "Husband/partner", "Respondent and husband/partner jointly" have been considered in the analysis.

The SLDHS 2016 contains a considerable number of questions, hence the obtained database consists of an extensive number of variables. Therefore, after a careful review and consideration of past literature, variables of interest that are related to the study were filtered and selected to examine. The associations between predictors (socio-background factors) and two responses of women's decision making were observed using chi-squared test. The predictors which are found to be significantly associated with responses (at 5\% level significance) were considered for modelling responses jointly with predictors. The important predictors identified using the chi-squared test are; the woman's sector (residence area), age, education, occupation, wealth index, marital status, number of children, ethnicity, religion, husband's education, husband's occupation and age gap between husband and wife.

It is obvious that the two responses regarding the decision making on household purchasing observed from the same respondent are likely to be correlated. Failure to account for such correlations by treating responses from the same respondents as independent may consequently yield incorrect inferences. Standard errors calculated by incorrectly assuming correlated observations to be independent tend to underestimate the true sampling variability, consequently yielding type I error of significance tests (Withanage et al. 2014). Hence, joint modelling of two outcomes provides better control over type I error rates in multiple tests and gains in efficiency in the parameter estimates.

The novelties of our research are 1) the correlation between the responses is considered into account while fitting the model using Generalized Linear Mixed Models approach, 2) all the possible outcomes of the responses were considered into analysis than merging into two outcomes (Acharya et al. 2010) and 3) parameter estimates in the model have odds ratio type interpretation after adjusting for other possible predictors. 


\subsection{Correlated Baseline Category Logit Model}

Denote the response vector for the $i^{\text {th }}(i=1,2, \cdots, n)$ subject by $\boldsymbol{Y}_{i}=\left(\boldsymbol{Y}_{i 1}^{\mathrm{T}}, \boldsymbol{Y}_{i 2}^{\mathrm{T}}\right)$ with $Y_{i 1}$ for Response 1 and $Y_{i 2}$ for Response 2. For the $j^{\text {th }}$ response $(j=1,2)$ of $i^{\text {th }}$ individual, $\boldsymbol{Y}_{i j}=\left(y_{i j 1}, y_{i j 2}, \cdots, y_{i j K}\right)^{\mathrm{T}}$ represent the multivariate trial, where

$$
y_{i j k}= \begin{cases}1 & \text { when the response is in category } k \\ 0 & \text { otherwise }\end{cases}
$$

Thus, $\sum_{k=1}^{K} y_{i j k}=1$ and $y_{i j K}=1-\left(y_{i j 1}+y_{i j 2}+\cdots+y_{i j, K-1}\right)$.

Let $\boldsymbol{x}_{i}=\left(x_{i 1}, x_{i 2}, \cdots, x_{i p}\right)^{\mathrm{T}}$ denote the predictor variables for subject $i$.

We assume a set of latent, random effects $b_{i}$, are shared by two responses of the same individual. This shared random intercept captures the unobserved factors specific to each individual which may influence the responses and tends account the correlation between the two responses of the same individual. The random intercepts are assumed to vary independently from one individual to another and be normally distributed with zero mean and constant variance, $\sigma_{b}^{2}$.

Let $\pi_{i j k}(\boldsymbol{x})=P\left(Y_{i j k}=k \mid \boldsymbol{x}\right)$ at fixed setting $\boldsymbol{x}$ for the explanatory variables, with $\sum_{k=1}^{K} \pi_{i j k}(x)=1$. For the observations at that setting, we treat the counts at the $K$ categories of $\boldsymbol{Y}_{i j}$ as multinomial with probabilities $\left\{\pi_{i j 1}(x), \pi_{i j 2}(x), \cdots, \pi_{i j K}(x)\right\}$.

Under the generalized linear mixed model setting, the baseline category logit model with random intercept, assuming the last level as the reference level, for the Response 1 is

$$
\log \left(\frac{\pi_{i 1 k}\left(x, b_{i}\right)}{\pi_{i 1 K}\left(x, b_{i}\right)}\right)=\beta_{01 k}+\boldsymbol{\beta}_{1 k}^{\mathrm{T}} \boldsymbol{x}_{i}+b_{i}, \quad k=1,2, \cdots, K-1,
$$

and similarly, for the Response 2 is

$$
\log \left(\frac{\pi_{i 2 k}\left(\boldsymbol{x}, b_{i}\right)}{\pi_{i 2 K}\left(\boldsymbol{x}, b_{i}\right)}\right)=\beta_{02 k}+\boldsymbol{\beta}_{2 k}^{\mathrm{T}} \boldsymbol{x}_{i}+b_{i}, \quad k=1,2, \cdots, K-1 .
$$

Given the shared random effect, $b_{i}$, it was assumed that $Y_{i 1}$ and $Y_{i 2}$ are independent. Hence, the marginal likelihood of $\left(Y_{i 1}, Y_{i 2}\right)$, for the $i^{\text {th }}$ individual can be obtained by integrating out the random effect $b_{i}$. By maximizing the likelihood (or log-likelihood) $\beta$ s can be estimated. However, this integral is usually intractable and some numerical or analytical approximation should be used. We adopted Gaussian quadrature method through PROC NLMIXED in SAS (codes are attached at the end).

\section{Result}

\subsection{Descriptive Statistics}

Of the respondents, 20.2 per cent and 64.7 per cent of ever-married women took decisions on their major household purchases alone and jointly with their husband, respectively (Table 1 ). This implies that nearly 85 per cent of ever-married women had participated in taking decisions on their major household purchases. For daily household purchases, 37.8 per cent and 48.7 per cent of ever-married 
women had participated in decision making alone and jointly with their husband, respectively. It is noted that, women alone taking decisions on daily household purchases is significantly higher than for their sole contribution on major household purchases. Cross-tabulation results show that socio-demographic characteristics are significantly associated with two types of women's decision making. The Table 1 summarizes how the percentages of the number of women vary on decisions on purchasing, based on their socio-demographic characteristics (rows).

Overall, it is clear that a majority of the decisions are made jointly among the respondent and her husband, between all characteristics for both decision types, which is a positive culmination. The participation in decision making increases with age, for both types of purchases. The percentages that the husband makes decisions approximately stay constant for this factor. Women in the estate sector have the least autonomous participation in decision making $(21.5 \%+61.5 \%=$ $83.0 \%$ in major household purchases decision making and $31.3 \%+52.4 \%=$ $83.7 \%$ in daily household purchases decision making) than the women in urban and rural sectors. It is noteworthy to report that women in estate sector too have more than $80 \%$ autonomy in decision making on household purchases. Urban women are more autonomous in major household purchasing (25.4\%) and rural women are more autonomous in daily household purchasing (38.3\%). Interestingly, as the level of education increases, the percentage of autonomous participation decreases and the percentage of join participation increases, which is again a satisfactory conclusion. This is the same with the wealth quintile results. With the increase of the number of children, the autonomous participation of the respondent making decisions increases, for both decision types. The join percentages decrease for both decision-making types for this factor. When considering major household purchases decision making, as the respondent works in a higher skill level occupation, her decision making autonomy increases, showing a decrease in joint participation and husband's autonomy as well. However, for daily household purchases decision making, women who are not working have a higher say $(35.6 \%)$ in decision making when compared to those who are employed in a low skill occupation (34.4\%). It again increases for medium and high skill level occupation groups. Interestingly, as the skill level of the husband's occupation increase, there is no significant rate of increase in husband's decision-making autonomy. In fact, it shows mixed variations. For major household purchases, the husbands employed in the high skill group have a higher percentage of autonomy (17\%) whereas it is the low skill occupation group for daily household purchases decision making (17\%). It is important to note that all the above interpretations are based on the marginal tables (two-way cross tabulations: response versus socio-demographic factor) and this interpretation may be misleading since many of socio-demographic factors are inter-correlated. This problem is overcome in the joint model since the parameter estimates are interpreted after adjusting for other socio-demographic factors in the model 
D. G. S. Chandradasa et al.

Table 1. Percentages of women decision making on major household purchases and daily household purchases by socio-demographic characteristics.

\begin{tabular}{|c|c|c|c|c|c|c|c|}
\hline & & \multicolumn{3}{|c|}{$\begin{array}{c}\text { Major household purchases } \\
\text { (row \%) }\end{array}$} & \multicolumn{3}{|c|}{$\begin{array}{c}\text { Daily household purchases } \\
\text { (row \%) }\end{array}$} \\
\hline & & $\begin{array}{l}\text { Respondent } \\
\text { alone }\end{array}$ & $\begin{array}{l}\text { Husband } \\
\text { alone }\end{array}$ & jointly & $\begin{array}{c}\text { Respondent } \\
\text { alone }\end{array}$ & $\begin{array}{c}\text { Husband } \\
\text { alone }\end{array}$ & jointly \\
\hline \multicolumn{2}{|c|}{ Overall } & 20.2 & 15.6 & 64.2 & 37.8 & 13.5 & 48.7 \\
\hline \multicolumn{8}{|c|}{ Classification by socio-demographic characteristics } \\
\hline \multirow{7}{*}{$\begin{array}{c}\text { Age } \\
\text { category }\end{array}$} & $15-20$ & 15.8 & 14.9 & 69.3 & 28.6 & 15.5 & 56.0 \\
\hline & $21-25$ & 16.0 & 15.7 & 68.3 & 29.9 & 14.8 & 55.3 \\
\hline & $26-30$ & 17.8 & 14.5 & 67.7 & 32.2 & 13.8 & 54.1 \\
\hline & $31-35$ & 21.6 & 13.7 & 64.7 & 37.1 & 13.5 & 49.4 \\
\hline & $36-40$ & 25.4 & 13.0 & 61.6 & 38.6 & 13.3 & 48.1 \\
\hline & $41-45$ & 29.0 & 13.7 & 57.3 & 42.2 & 13.2 & 44.7 \\
\hline & $46-49$ & 33.5 & 12.6 & 53.9 & 44.3 & 13.3 & 42.4 \\
\hline \multirow{3}{*}{ Sector } & Urban & 25.4 & 14.1 & 60.5 & 37.2 & 13.5 & 49.3 \\
\hline & Rural & 24.2 & 13.4 & 62.4 & 38.3 & 13.4 & 48.3 \\
\hline & Estate & 21.5 & 17.0 & 61.5 & 31.3 & 16.3 & 52.4 \\
\hline \multirow{4}{*}{ Education } & $\begin{array}{l}\text { Never attended } \\
\text { school }\end{array}$ & 41.5 & 17.6 & 40.9 & 47.2 & 16.6 & 36.2 \\
\hline & $\begin{array}{c}\text { Primary } \\
\text { education }\end{array}$ & 33.3 & 14.9 & 51.8 & 45.9 & 14.1 & 40.0 \\
\hline & $\begin{array}{l}\text { Secondary } \\
\text { education }\end{array}$ & 23.5 & 13.7 & 62.8 & 37.4 & 13.4 & 49.1 \\
\hline & Degree or above & 15.1 & 10.9 & 74.0 & 26.3 & 13.8 & 59.9 \\
\hline \multirow{3}{*}{$\begin{array}{l}\text { Wealth } \\
\text { quintile }\end{array}$} & Poor & 28.6 & 17.2 & 54.2 & 43.7 & 15.5 & 40.8 \\
\hline & Medium & 23.6 & 13.2 & 63.2 & 36.9 & 13.3 & 49.9 \\
\hline & Rich & 20.4 & 10.9 & 68.6 & 32.9 & 11.8 & 55.3 \\
\hline \multirow{4}{*}{$\begin{array}{l}\text { Number of } \\
\text { children }\end{array}$} & 0 & 17.6 & 14.4 & 68.0 & 28.2 & 13.8 & 58.0 \\
\hline & 1 to 2 & 24.4 & 13.0 & 62.6 & 38.1 & 13.1 & 48.8 \\
\hline & 3 to 4 & 25.7 & 14.7 & 59.6 & 39.6 & 14.4 & 46.0 \\
\hline & $5+$ & 29.9 & 17.9 & 52.2 & 45.7 & 14.8 & 39.4 \\
\hline \multirow{4}{*}{ Work } & Not working & 21.2 & 15.6 & 63.3 & 35.6 & 14.6 & 49.8 \\
\hline & Low skilled & 25.2 & 9.1 & 65.7 & 34.4 & 12.8 & 52.8 \\
\hline & Medium skilled & 30.1 & 10.0 & 59.9 & 42.3 & 10.6 & 47.1 \\
\hline & High skilled & 35.0 & 10.2 & 54.8 & 47.9 & 10.9 & 41.2 \\
\hline \multirow{4}{*}{$\begin{array}{l}\text { Husband's } \\
\text { occupation }\end{array}$} & Not working & 24.7 & 14.8 & 60.4 & 33.3 & 15.1 & 51.7 \\
\hline & Low skilled & 15.2 & 15.6 & 69.2 & 28.3 & 17.0 & 54.7 \\
\hline & Medium skilled & 15.6 & 13.5 & 70.8 & 28.1 & 14.4 & 57.5 \\
\hline & High skilled & 16.3 & 17.0 & 66.8 & 30.7 & 16.1 & 53.2 \\
\hline
\end{tabular}




\subsection{Analysis Based on the Joint Model}

The important predictors for the joint model were identified using the Chi-square test of association between the response and the predictor variable, and the predictors which are found to be significant at 10 per cent level were included to the model. This includes respondents' age, living area (sector), education level, occupation, wealth index, number of children, husband's occupation and the age gap between husband and wife.

For the joint model, define

- $\pi_{j 1}$ be the probability that the respondent alone making the decision for response $j(j=1,2)$,

- $\pi_{j 2}$ be the probability that the respondent's husband/partner alone making the decision for response $j(j=1,2)$,

- $\pi_{\beta}$ be the probability that the respondent and her husband/partner jointly making the decision for response $j(j=1,2)$.

Let us denote the predictor variables as; $X 1=$ Age, $X 2=$ Sector $(1=$ Urban, 2 $=$ Rural, $3=$ Estate), $X 3$ = Education $(1=$ Never attended school, $2=$ Primary education, $3=$ Secondary education, $4=$ Degree or above), $X 4=$ Wealth index $(1$ = Poor, $2=$ Medium, $3=$ Wealthy), $X 5=$ Number of children, $X 6=$ Respondents' occupation ( $1=$ Not working, 2 = Low skilled, $3=$ Medium skilled, $4=$ High skilled), $X 7=$ Husband/partner's occupation $(1=$ Not working, $2=$ Low skilled, $3=$ Medium skilled, $4=$ High skilled), $X 8=$ Age gap between the husband and wife.

Joint model (conditional on the random effect) for response 1 (Major household purchases decision making)

$$
\begin{aligned}
& \log \left(\frac{\pi_{11}}{\pi_{13}} \mid b_{i}\right)=-2.99+0.07 X 1-0.56 X 2(2)-1.81 X 2(3)-1.05 X 3(2) \\
& -1.29 \times 3(3)-2.03 \times 3(4)-0.87 X 4(2)-1.96 \times 4(3) \\
& +0.04 X 5-0.28 \times 6(2)-0.40 \times 6(3)-0.10 \times 6(4) \\
& +0.63 \times 7(2)+0.17 \times 7(3)+0.19 \times 7(4)+0.02 \times 8 \\
& \log \left(\frac{\pi_{12}}{\pi_{13}} \mid b_{i}\right)=-2.37+0.05 X 1-0.58 \times 2(2)-1.09 \times 2(3)-0.98 \times 3(2) \\
& -1.28 X 3(3)-1.45 X 3(4)-1.05 X 4(2)-2.12 X 4(3) \\
& +0.01 X 5+0.44 \times 6(2)-0.32 \times 6(3)+0.04 \times 6(4) \\
& +0.31 \times 7(2)+0.24 \times 7(3)-0.38 \times 7(4)+0.01 \times 8
\end{aligned}
$$

Joint model (conditional on the random effect) for response 2 (Daily household purchases decision making)

$$
\begin{aligned}
\log \left(\frac{\pi_{21}}{\pi_{23}} \mid b_{i}\right)= & -1.31+0.05 X 1-0.14 X 2(2)-2.09 X 2(3)+0.16 X 3(2) \\
& -0.13 X 3(3)-0.81 X 3(4)-1.06 X 4(2)-1.93 X 4(3)
\end{aligned}
$$




$$
\begin{aligned}
& +0.12 X 5-0.47 X 6(2)-0.77 X 6(3)-0.19 X 6(4) \\
& -0.04 X 7(2)+0.31 X 7(3)-0.25 \times 7(4)-0.004 X 8 \\
& \log \left(\frac{\pi_{22}}{\pi_{23}} \mid b_{i}\right)=-1.68+0.04 X 1-0.18 \times 2(2)-1.40 \times 2(3)-0.21 \times 3(2) \\
& -0.48 X 3(3)-0.74 X 3(4)-1.05 X 4(2)-1.97 X 4(3) \\
& +0.07 X 5+0.02 \times 6(2)-0.35 \times 6(3)-0.08 X 6(4) \\
& -0.13 \times 7(2)+0.42 \times 7(3)-0.29 \times 7(4)+0.01 \times 8
\end{aligned}
$$

Table 2 depicts the estimates and the relevant $P$-values of the joint model fitted for Response 1 and Response 2. Firstly we shall discuss the decision making regarding major household purchases. It is noted that following interpretations for the regression coefficients of the predictors are valid after controlling for

\begin{tabular}{|c|c|c|c|c|}
\hline \multirow{2}{*}{ Variable } & \multicolumn{2}{|c|}{$\begin{array}{c}\text { Response } 1 \\
\text { (Major household purchases } \\
\text { decision making) }\end{array}$} & \multicolumn{2}{|c|}{$\begin{array}{c}\text { Response } 2 \\
\text { (Daily household purchases } \\
\text { decision making) }\end{array}$} \\
\hline & $\begin{array}{c}\text { Model } 1 \\
\text { ( } P \text {-value) }\end{array}$ & $\begin{array}{c}\text { Model } 2 \\
\text { ( } p \text {-value) }\end{array}$ & $\begin{array}{l}\text { Model } 1 \\
\text { ( } p \text {-value) }\end{array}$ & $\begin{array}{c}\text { Model } 2 \\
(P \text {-value })\end{array}$ \\
\hline Intercept & $-2.99(<0.0001)$ & $-2.37(<0.0001)$ & $-1.31(0.0263)$ & $-1.68(0.0056)$ \\
\hline Age & $0.07(<0.0001)$ & $0.05(<0.0001)$ & $0.05(<0.0001)$ & $0.04(<0.0001)$ \\
\hline Sector (2) & $-0.56(0.0005)$ & $-0.58(0.0003)$ & $-0.14(0.3656)$ & $-0.18(0.2422)$ \\
\hline Sector (3) & $-1.81(<0.0001)$ & $-1.09(0.0004)$ & $-2.09(<0.0001)$ & $-1.40(<0.0001)$ \\
\hline Education (2) & $-1.05(0.0344)$ & $-0.98(0.05)$ & $0.16(0.7412)$ & $-0.21(0.6706)$ \\
\hline Education (3) & $-1.29(0.0058)$ & $-1.28(0.0067)$ & $-0.13(0.7787)$ & $-0.48(0.3059)$ \\
\hline Education (4) & $-2.03(0.0003)$ & $-1.45(0.0098)$ & $-0.81(0.1338)$ & $-0.74(0.1778)$ \\
\hline Wealth index (2) & $-0.87(<0.0001)$ & $-1.05(<0.0001)$ & $-1.06(<0.0001)$ & $-1.05(<0.0001)$ \\
\hline Wealth index (3) & $-1.96(<0.0001)$ & $-2.12(<0.0001)$ & $-1.93(<0.0001)$ & $-1.97(0.0001)$ \\
\hline Number of children & $0.04(0.4560)$ & $0.01(0.8158)$ & $0.12(0.0250)$ & $0.07(0.2229)$ \\
\hline Occupation (2) & $-0.28(0.1294)$ & $0.44(0.0234)$ & $-0.47(0.0100)$ & $0.02(0.9308)$ \\
\hline Occupation (3) & $-0.40(0.1683)$ & $-0.32(0.2929)$ & $-0.77(0.0054)$ & $-0.35(0.2278)$ \\
\hline Occupation (4) & $-0.10(0.6831)$ & $0.04(0.8557)$ & $-0.19(0.4198)$ & $-0.08(0.7529)$ \\
\hline Husband's occupation (2) & $0.63(0.0652)$ & $0.31(0.3775)$ & $-0.04(0.9094)$ & $-0.13(0.7113)$ \\
\hline Husband's occupation (3) & $0.17(0.2967)$ & $0.24(0.1474)$ & $0.31(0.0479)$ & $0.42(0.0088)$ \\
\hline Husband's occupation (4) & $0.19(0.1716)$ & $-0.38(0.0067)$ & $-0.25(0.0557)$ & $-0.29(-0.5551)$ \\
\hline Age gap & $0.02(0.07)$ & $0.01(0.4351)$ & $-0.004(0.7076)$ & $0.01(0.5895)$ \\
\hline
\end{tabular}
other predictors in the model and the given random effect. For the convenience of interpretation, this is not repeated for each but it should be understood. As age increases, there is still an increase in autonomy in decision making for the woman and husband/partner. This also is the same with the age gap, as the

Table 2. Parameter estimates ( $P$-value) of the correlated baseline category logit model. 
age gap increases, the women's autonomy increases. Women in urban sectors have more autonomy in decision making whereas women in rural and estate sectors take part in joint decision making. Women that belong to the medium and wealthy wealth quintiles take joint decision making compared to the poor quintile, implying the poor quintile is more autonomous. As the woman is educated, the couples tend to take joint decisions, when compared to those who aren't, except for women who are educated till the primary levels. For such women, the husband tends to be autonomous. When the wife is occupied in the low and high skill groups, the husband tends to have greater autonomy than women who are not employed. When she is employed in the medium skill group, they tend to make decisions jointly. When the husband is employed, the woman has greater autonomy in decision making, when compared to those of whose husbands aren't employed. When the number of children increases, she gains more autonomy.

Next, we shall discuss decision making regarding daily household purchases. As age increases, there is still an increase in autonomy in decision making for the woman. But with the age gap, as the age gap increases, the couples jointly make decisions. Women in urban sectors have more autonomy in decision making whereas women in rural and estate sectors take part in joint decision making. Women that belong to the medium and wealthy wealth quintiles take joint decision making compared to the poor quintile, implying the poor quintile is more autonomous. As the woman is educated, the couples tend to take joint decisions, when compared to those who aren't, except for women who are educated till the primary levels. For such women tends to be autonomous. When the wife is occupied in the low skill group, the husband tends to have greater autonomy than women who are not employed. When she is employed in the medium and high skill group, they tend to make decisions jointly. When the husband is employed in the low and high skill groups, the couple makes joint decisions, when compared to those of whose husbands aren't employed. When the number of children increases, she achieves more autonomy.

For the above correlated baseline category logit models, the baseline category was "the respondent and her husband making the decision jointly for the response". However, if the researcher is interested to know how women makes decision alone versus husband makes decision alone vary with their socio-economic background, our model has the flexibility to get the estimates using above models without refitting the model. This is followed by the fact that: $\log \left(\frac{\pi_{j 1}}{\pi_{j 2}}\right)=\log \left(\frac{\pi_{j 1}}{\pi_{j 3}}\right)-\log \left(\frac{\pi_{j 2}}{\pi_{j 3}}\right)$. Hence, the corresponding models for major household purchases decision making (response 1) and daily household purchases decision making (response 2) are given below respectively.

$$
\begin{aligned}
\log \left(\frac{\pi_{11}}{\pi_{12}} \mid b_{i}\right)= & -0.62+0.02 X 1+0.02 \times 2(2)-0.72 X 2(3)-0.07 X 3(2) \\
& -0.01 X 3(3)-0.58 X 3(4)+1.25 X 4(2)+0.16 X 4(3)
\end{aligned}
$$




$$
\begin{aligned}
+ & 0.03 X 5-0.72 X 6(2)-0.08 X 6(3)-0.14 X 6(4) \\
+ & 0.32 X 7(2)-0.07 X 7(3)+0.57 X 7(4)+0.01 X 8 \\
\log \left(\frac{\pi_{21}}{\pi_{22}} \mid b_{i}\right)= & 0.37+0.01 X 1+0.04 X 2(2)-0.69 X 2(3)+0.37 X 3(2) \\
& +0.35 X 3(3)-0.07 X 3(4)-0.01 X 4(2)+0.04 X 4(3) \\
& +0.05 X 5-0.49 X 6(2)-0.42 X 6(3)-0.11 X 6(4) \\
& +0.09 \times 7(2)-0.11 X 7(3)+0.04 X 7(4)-0.014 X 8
\end{aligned}
$$

\section{Conclusion}

\subsection{Discussion and Conclusion}

Study reveals that women's age, education status of the women and husband, number of children, residence area and economic status in the family emerged as important factors associated with women's autonomy in decision making. Increased age, well paid employment and having many living children are all positively associated with women's autonomy in decision making in both outcomes. Women from rural and estate areas are less likely be autonomous in decision making than the women from urban areas. Highly educated women are more likely to make decisions jointly but the women who never attended school are more likely to make decisions alone in both outcomes.

In this study, we applied correlated baseline category logit model to identify the influencing factors for women's decision making on daily household purchases and major household purchases. Modelling responses jointly is more appropriate than separate fitting of models that ignore the dependence between the decision making on daily household purchases and major household purchases. The Pearson Chi-square test of independence between the decision making on daily household purchases and major household purchases was highly significant $(P$-value $<0.0001)$ thereby indicating that the women who more autonomy in decision making on daily household purchases are more autonomy in decision making on major household purchases too. This was also concluded by our model since the variance of the random intercept was different from zero ( $\hat{\sigma}=4.4692, P$-value $<0.0001)$.

The study has several strengths compared to previous studies. First, the correlation between two responses is taken into consideration in our analytic approach thereby yielding unbiased estimates. Second, all the possible combinations of the response (respondent alone, respondent's husband alone or jointly) were considered into analysis than combining outcomes like respondent alone and respondents' husband. Though this makes models bit complicated, the researcher has more flexibility in reaching conclusions like for certain combination of predictor variables whether the respondent alone is more likely to make decisions or respondent's husband is more likely to make decisions than making decisions jointly. Third, our study covered a large population based-study, en- 
hancing its generalizability to all the married women in Sri Lanka. Fourth, the method we proposed can easily be extended to more than two responses. This can be done easily incorporating a vector of random effect instead of a single random effect in the joint model. That is, for $P$ number of responses, define $\boldsymbol{b}_{i}$ a $(P \times 1)$ random vector follows a multivariate normal distribution with zero mean vector and variance covariance matrix, $\boldsymbol{b}_{i} \sim N_{p}(\mathbf{0}, \boldsymbol{\Sigma})$.

\subsection{Limitation}

The major challenge of making this paper was the addressing all crucial sociological variations of study units. Since the study is based on the secondary data, it has been limited into some sociological patchiness such as age, age-gap, education level, occupational status and income level, size of the family and geographical position of household units only. Micro-level factors such as structural class ethics, the nature of interpersonal relationship among members of each family unit, the influences of neighborhood, kinship, and friendship networks and emotional factors such as religiosity, level of faithfulness of spouses that may be rather vitals in terms of household decision making proceedings. Therefore, the non-emphasizing of those internal mechanisms that determine family decision making process may be a significant limitation of this study. However, all vast-scale social researches have recorded attainable boundaries in their nature.

\section{Acknowledgements}

The authors gratefully acknowledge the support extended by the Department of Census of Statistics of Sri Lanka for providing the data necessary for the study, free of charge.

\section{Conflicts of Interest}

The authors declare no conflicts of interest regarding the publication of this paper.

\section{References}

Abraham, M. (1995). Ethnicity, Gender and Marital Violance: South Asian Women's Organizations in United States. Gender and Society, 9, 450-468. https://doi.org/10.1177/089124395009004004

Acharya, D., Bell, J., Simkhada, P., Van Teijlingen, E., \& Regmi, P. (2010). Women's Autonomy in Household Decision-Making: A Demographic Study in Nepal. Reproductive Health, 7, 1742-4755. https://doi.org/10.1186/1742-4755-7-15

Asian Development Bank (2019). Asian Development Bank, Country Gender Assessment Sri Lanka: An Update. https://www.adb.org

Bhopal, K. (2019). Gender, "Race" and Patriarchy: A Study of South Asian Women. London: Routledge. https://doi.org/10.4324/9780429456305

Boateng, G. O., Kuuire, V. Z., Ung, M., Amoyaw, J. A., Armah, F. A., \& Luginaah, I. (2014). Women's Empowerment in the Context of Millennium Development Goal 3: A Case Study of Married Women in Ghana. Social Indicators Research, 115, 137-158. https://doi.org/10.1007/s11205-012-0212-8 
CBSL Central Bank of Sri Lanka (2018). Central Bank Annual Report. Colombo.

Davis, H. L., \& Rigaux, B. P. (1974). Perception of Marital Roles in Decision Processes. Journal of Consumer Research, 1, 51-62. https://doi.org/10.1086/208581

Haque, S. E., Rahman, M., Mostofa, M. G., \& Zahan, M. S. (2012). Reproductive Health Care Utilization among Young Mothers in Bangladesh: Does Autonomy Matter? Women's Health Issues, 22, 71-80. https://doi.org/10.1016/j.whi.2011.08.004

Jejeebhoy, S., \& Sathar, Z. (2001). Women's Autonomy in India and Pakistan: The Influence of Religion and Region. Population and Development Review, 27, 687-712. https://doi.org/10.1111/j.1728-4457.2001.00687.x

McGinn, K. L., \& Oh, E. (2017). Gender, Social Class and Women's Employment. Current Opinion in Psychology, 18, 84-88. https://doi.org/10.1016/j.copsyc.2017.07.012

Mujahid-Mukhtar, E., Mukhtar, H., \& Abbink, G. (1991). Female Participation in Household Decision-Making: An Analysis of Consumer Durables' Acquisition in Pakistan [with Comments]. The Pakistan Development Review, 30, 953-964.

Nigatu, D., Gebremariam, A., Abera, M., Setegn, T., \& Deribe, K. (2014). Factors Associated with Women's Autonomy Regarding Maternal and Child Health Care Utilization in Bale Zone: A Community Based Cross-Sectional Study. BMC Women's Health, 14, 79. https://doi.org/10.1186/1472-6874-14-79

Pew Research Centre (PRC) (2016). Religion and Public Life. The Gender Gap in Religion around the World.

Senarath, U., \& Gunawardena, N. S. (2009). Women's Autonomy in Decision Making for Health Care in South Asia. Asia Pacific Journal of Public Health, 21, 137-143. https://doi.org/10.1177/1010539509331590

Smyth, E. (2007). Gender and Education. In R. Teese, S. Lamb, M. Duru-Bellat, \& S. Helme (Eds.), International Studies in Educational Inequality, Theory and Policy (135-153). Dordrecht: Springer.

The DHS Program (2016). The DHS Program-Quality Information to Plan, Monitor and Improve Population, Health, and Nutrition Programs.

http://www.statistics.gov.lk/Health/StaticalInformation/DemographicAndHealthSurve y-2016FullReport

UNDP (2016). Human Development, Disparity and Vulnerability: Women in South Asia. UNDP Human Development Report, Background Paper.

http://hdr.undp.org/en/content/human-development-disparity-and-vulnerability-wom en-south-asia

Withanage, N., de Leon, A. R., \& Rudnisky, C. (2014). Joint Estimation of Disease-Specific Sensitivities and Specificities in Reader-Based Multi-Disease Diagnostic Studies of Paired Organs. Journal of Applied Statistics, 41, 2282-2297.

https://doi.org/10.1080/02664763.2014.909790

Xia, Y., Ahmed, Z. U., Ghingold, M., Hwa, N. K., Li, T. W., \& Ying, W. T. C. (2016). Spousal Influence in Singaporean Family Purchasing Decision-Making Process: A Cross Cultural Comparison. Asia-Pacific Journal of Marketing and Logistics, 18, 201-222. https://doi.org/10.1108/13555850610675661

Yusof, S. A. (2015). Household Decision-Making in Malaysia: The Ethnic Dimension. Social Indicators Research, 124, 283-293. https://doi.org/10.1007/s11205-014-0782-8

Yusof, S. A., \& Duasa, J. (2010). Household-Decision Making and Expenditure Patterns of Married Men and Women in Malaysia. Journal Family and Economic Issues, 31, 371-381. https://doi.org/10.1007/s10834-010-9200-9 


\section{Appendix}

The SAS code of which the joint modelling was performed is as follows.

proc nlmixed qpoints $=20$ technique $=$ newrap data $=$ sample3;

parms

beta $\_110=-2.1$ beta $\_111=0.03$ beta $\_112=0.01$ beta $\_113=-0.18$ beta $\_114=-0.81$ beta $\_115=-0.27$ beta $\_116=-0.7$

beta_117 $=0.06$ beta_118 $=0.22$ beta_119 $=0.3$ beta_1110 $=-0.3$ beta_1111 $=-0.34$ beta_1112 $=-0.3$ beta_1113 $=0.03$

beta_1114 $=-0.40$ beta_1115 $=-0.48$ beta_1116 $=-0.89$

beta $\_120=-1.1$ beta $\_121=0.01$ beta $\_122=-0.001$ beta $-123=-0.22$ beta $-124=-0.11$ beta $\_125=-0.43$ beta $126=-0.8$

beta $\_127=-0.4$ beta $\_128=-0.33$ beta_129 $=-0.4$ beta $\_1210=0.1$ beta $1211=-0.2$ beta $-1212=0.02$ beta $1213=0.001$

beta_1214 $=-0.27$ beta_1215 $=-0.38$ beta_1216 $=-0.27$

beta $\_210=-1.04$ beta $\_211=0.01$ beta $\_212=-0.002$ beta $\_213=0.04$ beta $\_214=-0.86$ beta $\_215=-0.37$ beta $\_216=-0.68$

beta_217 $=-0.17$ beta $\_218=-0.14$ beta_219 $=0.24$ beta_2110 $=0.06$ beta_2111 $=-0.07$ beta_2112 $=-0.0005$ beta_2113 $=0.05$

beta $2114=0.12$ beta_2115 $=0.025$ beta_2116 $=-0.31$

beta $\_220=-1.36$ beta $\_221=0.01$ beta $\_222=0.007$ beta $\_223=-0.07$ beta $\_224=-0.14$ beta $\_225=-0.35$ beta $\_226=-0.68$

beta $\_227=-0.12$ beta $\_228=-0.19$ beta $\_229=-0.2$ beta $\_2210=0.27$ beta $\_2211=-0.03$ beta $\_2212=-0.09$ beta $\_2213=0.01$

beta $\_2214=-0.19$ beta $\_2215=-0.26$ beta $\_2216=-0.17 \mathrm{~s} 1=5$;

if $\mathrm{Y}=$ "y1" then do;

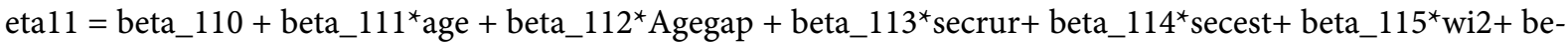

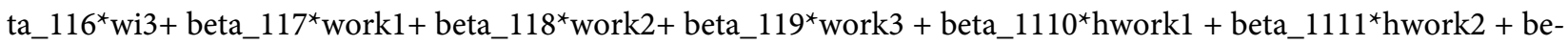

ta_1112*hwork $3+$ beta_ $1113^{*}$ Children + beta_ $1114^{*}$ edu $1+$ beta_1115*edu $2+$ beta_1116*edu $3+\mathrm{u}$;

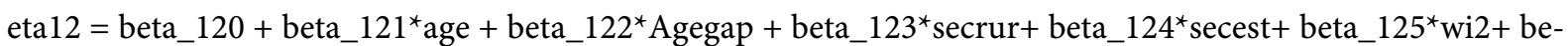

ta_126*wi3+ beta_127*work1+ beta_128*work2+ beta_129* work $3+$ beta_1210*hwork1 + beta_1211*hwork $2+$ be-

ta_1212*hwork $3+$ beta_ $1213^{*}$ Children + beta_ $1214^{*}$ edu $1+$ beta_ $1215^{\star}$ edu $2+$ beta_1216*edu $3+\mathrm{u}$;

$\mathrm{ll}=\mathrm{y}_{-} \mathrm{i} 1^{*} \mathrm{eta} 11+\mathrm{y} \_\mathrm{i} 2^{*} \operatorname{eta} 12-\log (1+\exp (\operatorname{eta} 11)+\exp (\operatorname{eta} 12))$;

end;

if $\mathrm{Y}=$ "y2" then do;

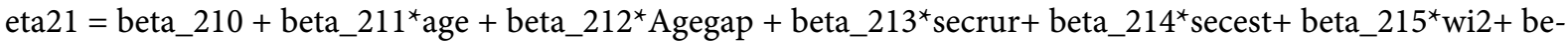

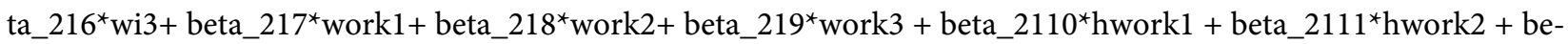

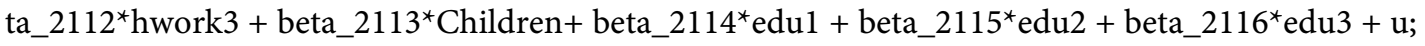

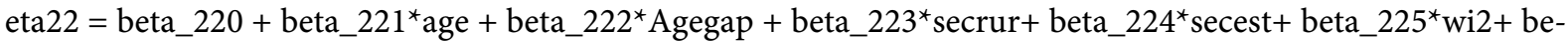

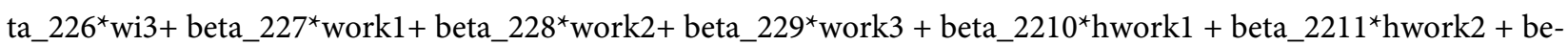

ta $\_2212^{*}$ hwork $3+$ beta $\_213^{*}$ Children + beta $\_214^{*}$ edu $1+$ beta $\_215^{*}$ edu $2+$ beta $\_2216^{*}$ edu $3+$ u;

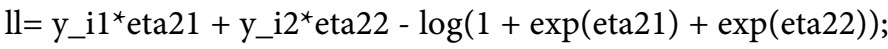

end;

model zz general(ll);

random $\mathrm{u} \sim \operatorname{normal}\left(0, \mathrm{~s} 1^{\star} \mathrm{s} 1\right)$ subject=id;

run; 